
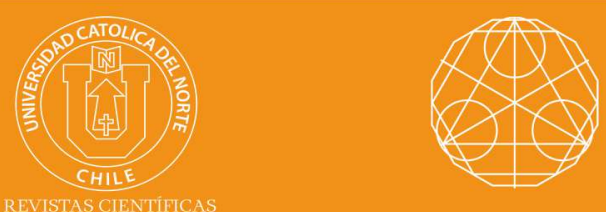

\title{
Discussion on relation-theoretic for $J S$-quasi-contractions of uni/multi-dimensional mappings with the transitivity
}

Kanokwan Sawangsup ${ }^{1}$ (1) orcid.org/0000-0003-4291-5228 Wutiphol Sintunavarat ${ }^{2}$ (1) orcid.org/0000-0002-0932-1332

Thammasat University, Dept. of Mathematics and Statistics, Rangsit, TH-13, Thailand

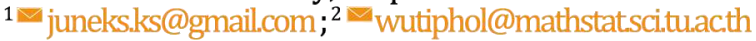

\section{Abstract:}

We introduce the notion of a JS $S_{\mathfrak{i}}$ quasi-contraction mapping, where $\mathbb{l}$ is a binary relation on its domain. Also, we prove some fixed point results for such contractions in complete metric spaces endowed with a transitive relation. An example is given to substantiate our obtained theorems. In addition, we introduce a $J S_{\mathfrak{i n}}$-quasicontraction and also establish fixed point of $\mathrm{N}$-order theorems for such contractions.

Keywords: Transitive relation; Fixed point of N-order

$\operatorname{MSC}(2010): 47 \mathrm{H} 10,54 \mathrm{H} 25$.

\section{Cite this article as (IEEE citation style):}

K. Sawangsup and W. Sintunavarat, "Discussion on relationtheoretic for JS-quasi-contractions of uni/multi-dimensional mappings with the transitivity", Proyecciones (Antofagasta, On line), vol. 39, no. 3, pp. 559-580, Jun. 2020, doi: 10.22199/issn.0717-6279-2020-03-0034.

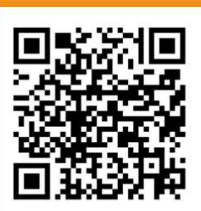

Article copyright: (C) 2020 Kanokwan Sawangsup and Wutiphol Sintunavarat. This is an open access article distributed under the terms of the Creative Commons Licence, which permits unrestricted use and distribution provided the original author and source are credited

(cc) BY 


\section{Introduction}

The fundamental result of metric fixed point theory is the Banach contraction principle, which is due to the great Polish mathematician Banach in his thesis [1]. This principle states that for a given complete metric space $(X, d)$, a self mapping $T$ on $X$ satisfying the condition that $d(T x, T y) \leq d(x, y)$ for all $x, y \in X$, where $k \in[0,1)$, has a unique fixed point. Many mathematicians have extended the Banach contraction principle in various directions. For instance, the popular extension related to relations on the domain of a mappings in a fixed point result was given by Ran and Reurings [2] in 2003. They proved fixed points results in partially ordered metric spaces and presented some applications to matrix equations. Based on the utility of this direction, Alam and Imdad [3] presented a new extension of the classical Banach contraction principle to metric spaces endowed with a universal binary relation which is weaker than a partially ordered metric space. One of other directions is an extension of the dimension of the domain of a mapping in fixed point results from one dimension to multi-dimension. The first research in this direction is investigated in 1987 by Guo and Lakshmikantham [4]. They first introduced the notion of a coupled fixed point. Subsequently, Bhaskar and Lakshmikantham [5] defined the concept of the mixed monotone property and used it to prove the coupled fixed point theorems in partially ordered metric spaces. In [6], Asgari and Mousavi discussed some results on the existence and uniqueness of a coupled fixed point in metric spaces endowed with a reflexive relation and presented some applications to matrix equations. In 2010, Samet and Vetro [7] introduced the notion of fixed point of $N$-order as natural extension of the notion of coupled fixed point and they also established results for fixed point of $N$-order in complete metric spaces.

One of the most interesting generalizations of the Banach contraction principle is focused on the contractive condition. Here, some famous fixed point results in this trend are given. In [8], Wardowski introduced the concept of an $F$-contraction and proved some fixed point results by considering this new family of contractions. At the same year, Khojasteha et al. [9] first introduced the definition of a simulation function and defined a new contraction namely $\mathcal{Z}$-contraction with respect to a simulation function and establish fixed point results for such contractions. In 2014, Jleli and Samet [12] defined the new contraction namely $J S$-contraction and also investigated the existence and uniqueness of a fixed point for $J S$ contractions. In 2015, Hussain et al. [13] presented the extension of fixed 
point results for Ćirić contraction due to Ćirić $[10,11]$ and fixed point results for $J S$-contractions. One year later, Li and Jiang [14] introduced the concept of a $J S$-quasi-contraction that the involving control function is continuous and nondecreasing, and they also proved a fixed point result for such contractions.

The aim of this paper is to introduce the concept a new contraction

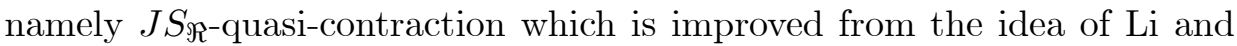
Jiang [14] under a special relation which is weaker than a partial order. We also establish fixed point results for $J s_{\Re}$-quasi-contraction mappings in complete metric spaces endowed with a transitive relation and provide an example supporting our main result. In addition, we present new fixed point of $N$-order results for $J S_{\Re^{N} \text {-contractions in metric spaces endowed }}$ with a transitive relation.

\section{Preliminaries}

We start our consideration by giving a brief review of definitions and basic properties of binary relations. Henceforth, $X, \mathbf{R}^{+}=(0, \infty), \mathbf{N}$ and $\mathbf{N}_{0}=$ $\mathbf{N} \cup\{0\}$ denote a nonempty set, the set of positive real numbers, the set of positive integers and the set of nonnegative integers, respectively.

For convenience, the notion $\Psi$ is denoted by a family of all functions $\psi:(0, \infty) \rightarrow(1, \infty)$ satisfies the following properties:

- $\psi$ is continuous;

- $\psi$ is nondecreasing.

The following definition is the concept of a $J S$-quasi-contraction, which was introduced by Li and Jiang [14].

Definition 2.1 ([14]). Let $(X, d)$ be a metric space. A mapping $T: X \rightarrow$ $X$ is said to be a $J S$-quasi-contraction if there exist a function $\psi:(0, \infty) \rightarrow$ $(1, \infty)$ and $\lambda \in(0,1)$ such that

$$
\psi(d(T x, T y)) \leq\left[\psi\left(M_{d}(x, y)\right)\right]^{\lambda}
$$

for all $x, y \in X$ with $T x \neq T y$, where

$$
M_{d}(x, y)=\max \left\{d(x, y), d(x, T x), d(y, T y), \frac{d(x, T y)+d(y, T x)}{2}\right\} .
$$


They also proved the following fixed point result for $J S$-quasi-contraction mapping, which is the motivation to write this paper.

Theorem 2.2 ([14]). Let $(X, d)$ be a complete metric space. Then a JSquasi-contraction mapping $T: X \rightarrow X$ has a unique fixed point in $X$.

One of our main aims is to extend the previous result to the multidimensional case involving a kind of a binary relation. Next, we now give some preliminaries in this sense.

Definition $2.3([\mathbf{1 5}])$. A binary relation on a nonempty set $X$ is a nonempty subset $\Re$ of $X \times X$. If $(x, y) \in \Re$, we also denote it by $x \Re y$, and we say that $x$ is $\Re$-related to $y$.

Definition 2.4. The binary relation $\Re$ on a nonempty set $X$ is transitive if $(x, z) \in \Re$ for all $x, y, z \in X$ with $(x, y) \in \Re$ and $(y, z) \in \Re$.

Definition 2.5 ([3]). Let $X$ be a nonempty set. Given a mapping $T$ : $X \rightarrow X$, a binary relation $\Re$ defined on $X$ is called $T$-closed if for any $x, y \in X,(x, y) \in \Re \Rightarrow(T x, T y) \in \Re$.

The property in the above definition is equivalent to say that $T$ is $\Re$ nondecreasing (see in [17]).

Definition 2.6 ([16]). Let $\Re$ be a binary relation on a nonempty set $X$. For each $x, y \in X$, a path of length $k$, where $k$ is a natural number, in $\Re$ from $x$ to $y$ is a finite sequence $\left\{z_{0}, z_{1}, z_{2}, \ldots, z_{k}\right\} \subseteq X$ satisfying the following conditions:

(i) $z_{0}=x$ and $z_{k}=y$;

(ii) $\left(z_{i}, z_{i+1}\right) \in \Re$ for all $i \in\{0,1,2, \ldots, k-1\}$.

We denote by $\Upsilon(x, y, \Re)$ the family of all paths in $\Re$ from $x$ to $y$.

Note that a path of length $k$ involves $k+1$ elements of $X$, although they are not necessarily distinct. 
Definition $2.7([\mathbf{1 7}])$. A metric space $(X, d)$ endowed with a binary relation $\Re$ is $\Re$-nondecreasing-regular if for all sequence $\left\{x_{n}\right\} \subseteq X$, the following condition holds:

$$
\left.\begin{array}{c}
\left(x_{n}, x_{n+1}\right) \in \Re, \quad \forall n \in \mathbf{N}, \\
x_{n} \rightarrow u \in X
\end{array}\right\} \Rightarrow\left(x_{n}, u\right) \in \Re, \quad \forall n \in \mathbf{N} .
$$

Throughout this paper, for a given binary relation $\Re$ on a nonempty set $X$ and a given mapping $T: X \rightarrow X$, let us denote by $\operatorname{Fix}(T)$ and $X(T ; \Re)$ a set of all fixed points of $T$ and the set of all points $x \in X$ satisfying $(x, T x) \in \Re$, respectively, that is,

$$
\operatorname{Fix}(T):=\{z \in X: z=T z\} \text { and } X(T ; \Re):=\{x \in X:(x, T x) \in \Re\}
$$

\section{Main results}

In this section, inspired by the notion of a $J S$-quasi-contraction, we introduce the new kind of self-mappings on a metric space involving a binary relation as follows.

Definition 3.1. Given a metric space $(X, d)$ and a binary relation $\Re$ on $X$, let

$$
\mathcal{Z}=\{(x, y) \in \Re: T x \neq T y\} .
$$

A self-mapping $T$ on $X$ is called a $J S_{\Re \text {-quasi-contraction if there exist a }}$ function $\psi:(0, \infty) \rightarrow(1, \infty)$ and $\lambda \in(0,1)$ such that

$$
\psi(d(T x, T y)) \leq\left[\psi\left(M_{d}(x, y)\right)\right]^{\lambda}
$$

$$
\text { for all }(x, y) \in \mathcal{Z} \text {, where } M_{d}(x, y)=\max \left\{d(x, y), d(x, T x), d(y, T y), \frac{d(x, T y)+d(y, T x)}{2}\right\} \text {. }
$$

One of the main advantages of the following results is the fact that a focused relation is weaker than a partial order. It uses only a transitivity property to guarantee the existence and uniqueness of a fixed point of $J S_{\Re \text {-quasi- }}$ contraction mappings.

Theorem 3.2. Let $(X, d)$ be a complete metric space, $\Re$ a transitive relation on $X$, and $T$ a self-mapping on $X$. Suppose that the following conditions hold:

(a) $X(T ; \Re)$ is nonempty, 
(b) $\Re$ is $T$-closed,

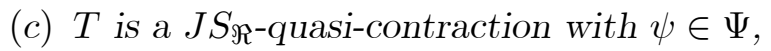

(d) $T$ is continuous.

Then $T$ has a fixed point. Moreover, for each $x_{0} \in X(T ; \Re)$, the Picard sequence $\left\{T^{n} x_{0}\right\}$ converges to a fixed point of $T$.

Proof. Let $x_{0}$ be an arbitrary point in $X(T ; \Re)$. Put $x_{n}=T^{n} x_{0}$ for all $n \in \mathbf{N}_{0}$. If $x_{n^{*}}=x_{n^{*}+1}$ for some $n^{*} \in \mathbf{N}_{0}$, then $x_{n^{*}}$ is a fixed point of $T$ and the proof is completed. Next, we suppose that $x_{n} \neq x_{n+1}$ for all $n \in \mathbf{N}_{0}$ and so $T x_{n} \neq T x_{n+1}$ for all $n \in \mathbf{N}_{0}$. Since $\left(x_{0}, T x_{0}\right) \in \Re$, using that $\Re$ is $T$-closed, we get

$$
\left(x_{n}, x_{n+1}\right) \in \Re
$$

for all $n \in \mathbf{N}_{0}$. Thus,

$$
\left(x_{n}, x_{n+1}\right) \in \mathcal{Z}
$$

for all $n \in \mathbf{N}_{0}$. Since

$$
\begin{aligned}
& \max \left\{d\left(x_{n}, x_{n+1}\right), d\left(x_{n+1}, x_{n+2}\right)\right\} \\
\leq & \max \left\{d\left(x_{n}, x_{n+1}\right), d\left(x_{n+1}, x_{n+2}\right), \frac{d\left(x_{n}, x_{n+2}\right)+d\left(x_{n+1}, x_{n+1}\right)}{2}\right\} \\
= & M_{d}\left(x_{n}, x_{n+1}\right) \\
\leq & \max \left\{d\left(x_{n}, x_{n+1}\right), d\left(x_{n+1}, x_{n+2}\right), \frac{d\left(x_{n}, x_{n+1}\right)+d\left(x_{n+1}, x_{n+2}\right)}{2}\right\} \\
= & \max \left\{d\left(x_{n}, x_{n+1}\right), d\left(x_{n+1}, x_{n+2}\right)\right\}
\end{aligned}
$$

for all $n \in \mathbf{N}_{0}$, we obtain $M_{d}\left(x_{n}, x_{n+1}\right)=\max \left\{d\left(x_{n}, x_{n+1}\right), d\left(x_{n+1}, x_{n+2}\right)\right\}$

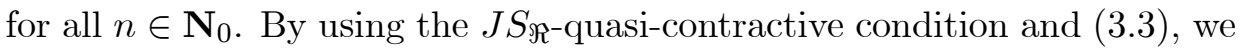
get

$$
\begin{aligned}
\psi\left(d\left(x_{n+1}, x_{n+2}\right)\right) & =\psi\left(d\left(T x_{n}, T x_{n+1}\right)\right) \\
& \leq\left[\psi\left(M_{d}\left(x_{n}, x_{n+1}\right)\right)\right]^{\lambda} \\
& =\left[\psi\left(\max \left\{d\left(x_{n}, x_{n+1}\right), d\left(x_{n+1}, x_{n+2}\right)\right\}\right)\right]^{\lambda}
\end{aligned}
$$


for all $n \in \mathbf{N}_{0}$. Assume that there exists $p_{0} \in \mathbf{N}_{0}$ such that $d\left(x_{p_{0}+1}, x_{p_{0}+2}\right)>$ $d\left(x_{p_{0}}, x_{p_{0}+1}\right)$. Thus,

$$
\begin{aligned}
\psi\left(d\left(x_{p_{0}+1}, x_{p_{0}+2}\right)\right) & \leq\left[\psi\left(d\left(x_{p_{0}+1}, x_{p_{0}+2}\right)\right)\right]^{\lambda} \\
& <\psi\left(d\left(x_{p_{0}+1}, x_{p_{0}+2}\right)\right)
\end{aligned}
$$

which is a contradiction. Therefore,

$$
d\left(x_{n+1}, x_{n+2}\right) \leq d\left(x_{n}, x_{n+1}\right)
$$

for all $n \in \mathbf{N}_{0}$. Hence $\left\{d\left(x_{n}, x_{n+1}\right)\right\}$ is a nonincreasing sequence and bounded below. Then there exists $\beta \geq 0$ such that

$$
\lim _{n \rightarrow \infty} d\left(x_{n}, x_{n+1}\right)=\beta
$$

and

$$
d\left(x_{n}, x_{n+1}\right) \geq \beta
$$

for all $n \in \mathbf{N}_{0}$. Next, we will show that $\beta=0$. Suppose that $\beta>0$. Since $\psi$ is nondecreasing, using (3.3), (3.4), and (3.6), we get

$$
\begin{aligned}
1 & <\psi(\beta) \\
& \leq \psi\left(d\left(x_{n}, x_{n+1}\right)\right) \\
& \leq\left[\psi\left(d\left(x_{n-1}, x_{n}\right)\right)\right]^{\lambda} \\
& \vdots \\
& \leq\left[\psi\left(d\left(x_{0}, x_{1}\right)\right)\right]^{\lambda^{n}}
\end{aligned}
$$

for all $n \in \mathbf{N}_{0}$. Taking $n \rightarrow \infty$ in (3.7), we obtain $1<\psi(\beta) \leq 1$, which is a contradiction. Therefore, $\beta=0$ and so

$$
\lim _{n \rightarrow \infty} d\left(x_{n}, x_{n+1}\right)=0 .
$$

Next, we will show that $\left\{x_{n}\right\}$ is a Cauchy sequence in $X$. Assume that $\left\{x_{n}\right\}$ is not a Cauchy sequence. Thus, there exists $\epsilon>0$ and two subsequences $\left\{x_{n_{k}}\right\}$ and $\left\{x_{m_{k}}\right\}$ of $\left\{x_{n}\right\}$ such that

$$
d\left(x_{n_{k}}, x_{m_{k}}\right) \geq \epsilon
$$


for all $m_{k}>n_{k}>k$. Let $m_{k}$ be the smallest number with respect to $n_{k}$ such that (3.9) holds. It follows that

$$
d\left(x_{n_{k}}, x_{m_{k}-1}\right)<\epsilon .
$$

From (3.9) and (3.10), we get

$$
\begin{aligned}
\epsilon & \leq d\left(x_{n_{k}}, x_{m_{k}}\right) \\
& \leq d\left(x_{n_{k}}, x_{m_{k}-1}\right)+d\left(x_{m_{k}-1}, x_{m_{k}}\right) \\
& \leq \epsilon+d\left(x_{m_{k}-1}, x_{m_{k}}\right) .
\end{aligned}
$$

Taking $k \rightarrow \infty$ in (3.11) and using (3.8), we obtain

$$
\lim _{k \rightarrow \infty} d\left(x_{n_{k}}, x_{m_{k}}\right)=\epsilon .
$$

By the triangle inequality, we get

$$
\begin{aligned}
& d\left(x_{n_{k}+1}, x_{m_{k}+1}\right) \leq d\left(x_{n_{k}+1}, x_{n_{k}}\right)+d\left(x_{n_{k}}, x_{m_{k}}\right)+d\left(x_{m_{k}}, x_{m_{k}+1}\right), \\
& d\left(x_{n_{k}}, x_{m_{k}}\right) \leq d\left(x_{n_{k}}, x_{n_{k}+1}\right)+d\left(x_{n_{k}+1}, x_{m_{k}+1}\right)+d\left(x_{m_{k}+1}, x_{m_{k}}\right) .
\end{aligned}
$$

Letting $k \rightarrow \infty$ in (3.13) and (3.14), using (3.8) and (3.12), we get

$$
\lim _{k \rightarrow \infty} d\left(x_{n_{k}+1} \cdot x_{m_{k}+1}\right)=\epsilon
$$

By using the above process, we get

$$
\lim _{k \rightarrow \infty} d\left(x_{n_{k}}, x_{m_{k}+1}\right)=\lim _{k \rightarrow \infty} d\left(x_{n_{k}+1}, x_{m_{k}}\right)=\epsilon .
$$

Now, we have

$$
\begin{aligned}
\epsilon & \leq d\left(x_{n_{k}}, x_{m_{k}}\right) \\
& \leq M_{d}\left(x_{n_{k}}, x_{m_{k}}\right) \\
& =\max \left\{d\left(x_{n_{k}}, x_{m_{k}}\right), d\left(x_{n_{k}}, x_{n_{k}+1}\right), d\left(x_{m_{k}}, x_{m_{k}+1}\right), \frac{d\left(x_{n_{k}}, x_{m_{k}+1}\right)+d\left(x_{n_{k}+1}, x_{m_{k}}\right)}{2}\right\} .
\end{aligned}
$$

By (3.8), (3.12), (3.15) and (3.16), we get

$$
\lim _{n \rightarrow \infty} M_{d}\left(x_{n_{k}}, x_{m_{k}}\right)=\epsilon
$$


This follows from (3.15) and (3.16) that there exists a positive integer $k_{0}$ such that $d\left(x_{n_{k}+1}, x_{m_{k}+1}\right)>0$ and $M_{d}\left(x_{n_{k}}, x_{m_{k}}\right)>0$ for all $k \geq k_{0}$. Since $m_{k}>n_{k}$ and $\Re$ is a transitive relation, we have $\left(x_{n_{k}}, x_{m_{k}}\right) \in \Re$ and so

$$
\begin{aligned}
\psi\left(d\left(x_{n_{k}+1}, x_{m_{k}+1}\right)\right) & =\psi\left(d\left(T x_{n_{k}}, T x_{m_{k}}\right)\right) \\
& \leq\left[\psi\left(d\left(x_{n_{k}}, x_{m_{k}}\right)\right)\right]^{\lambda}
\end{aligned}
$$

for all $m_{k}>n_{k}>k_{0}$. Letting $k \rightarrow \infty$ in (3.18), by (3.15), (3.17), and the continuity of $\psi$, we get

$$
\begin{aligned}
\psi(\epsilon) & =\lim _{k \rightarrow \infty} \psi\left(d\left(x_{n_{k}+1}, x_{m_{k}+1}\right)\right) \\
& \leq \lim _{k \rightarrow \infty}\left[\psi\left(M_{d}\left(x_{n_{k}}, x_{m_{k}}\right)\right)\right]^{\lambda} \\
& =[\psi(\epsilon)]^{\lambda} \\
& <\psi(\epsilon),
\end{aligned}
$$

which is a contradiction. Therefore, $\left\{x_{n}\right\}$ is a Cauchy sequence in $X$. Since $X$ is a complete metric space, there exists $x^{*} \in X$ such that $x_{n} \rightarrow x^{*}$ as $n \rightarrow \infty$. By the continuity of $T$, we get $T x^{*}=x^{*}$. This completes the proof.

The following example shows the usability of Theorem 3.2.

Example 3.3. Let $X=[0, \infty)$ and $d: X \times X \rightarrow[0, \infty)$ be the Euclidean metric defined by $d(x, y)=|x-y|$ for all $x, y \in X$. Thus $(X, d)$ is a complete metric space. Consider a sequence $\left\{\phi_{n}\right\}_{n \in \mathbf{N}}$ defined as

$$
\phi_{n}=\frac{n(n+1)(n+2)}{3} \quad \text { for all } n \in \mathbf{N}
$$

Define a binary relation $\Re$ on $X$ by

$$
\Re:=\left\{\left(\phi_{i}, \phi_{j}\right): i, j \in \mathbf{N} \text { with } i>j\right\} \cup\left\{\left(\phi_{k}, 1\right): k \in \mathbf{N}\right\} \cup\{(1,1)\} .
$$

Thus, $\Re$ is a transitive relation. Now, we define a mapping $T: X \rightarrow X$ by 


$$
T x= \begin{cases}x & \text { if } 0 \leq x \leq 1, \\ 1 & \text { if } 1 \leq x \leq \phi_{1}, \\ 1+\left(\frac{\phi_{1}-1}{\phi_{2}-\phi_{1}}\right)\left(x-\phi_{1}\right) & \text { if } \phi_{1} \leq x \leq \phi_{2}, \\ \phi_{1}+\left(\frac{\phi_{2}-\phi_{1}}{\phi_{3}-\phi_{2}}\right)\left(x-\phi_{2}\right) & \text { if } \phi_{2} \leq x \leq \phi_{3}, \\ \phi_{2}+\left(\frac{\phi_{3}-\phi_{2}}{\phi_{4}-\phi_{3}}\right)\left(x-\phi_{3}\right) & \text { if } \phi_{3} \leq x \leq \phi_{4}, \\ \vdots & \vdots \\ \phi_{n-1}+\left(\frac{\phi_{n}-\phi_{n-1}}{\phi_{n+1}-\phi_{n}}\right)\left(x-\phi_{n}\right) & \text { if } \phi_{n} \leq x \leq \phi_{n+1}, \\ \vdots & \vdots\end{cases}
$$

It is easy to see that $T$ is continuous (see Figure 1 ) and $\Re$ is $T$-closed. Next, we will show that $T$ is a $J S_{\Re \text {-quasi-contraction mapping with a function }}$ $\psi \in \Psi$ defined by $\psi(t)=e^{t e^{t}}$ for all $t>0$. It suffices to show that there exists $\lambda \in(0,1)$ such that for each $(x, y) \in \mathcal{Z}=\{(x, y) \in \Re: T x \neq T y\}$, we have

$$
\frac{d(T x, T y) e^{d(T x, T y)-M_{d}(x, y)}}{M_{d}(x, y)} \leq \lambda .
$$

So, let $(x, y) \in \mathcal{Z}=\{(x, y) \in \Re: T x \neq T y\}$. This implies that $(x, y)=$ $\left(\phi_{i}, \phi_{j}\right)$ for all $i, j \in \mathbf{N}$ with $i>j$ or $(x, y)=\left(\phi_{k}, 1\right)$ for all $k \in \mathbf{N} \backslash\{1\}$.

Case I: We consider $(x, y)=\left(\phi_{i}, \phi_{j}\right)$ for all $i, j \in \mathbf{N}$ with $i>j$. If $i>j>1$, then

$$
\begin{aligned}
d(T x, T y) & =d\left(T \phi_{i}, T \phi_{j}\right) \\
& =d\left(\phi_{i-1}, \phi_{j-1}\right) \\
& =\left|\frac{(i-1) i(i+1)-(j-1) j(j+1)}{3}\right| \\
& =\frac{(i-j)\left(i^{2}+j^{2}+i j-1\right)}{3}
\end{aligned}
$$

and

$M_{d}(x, y)=M_{d}\left(\phi_{i}, \phi_{j}\right)$ 
Fixed points and diametral sets for sequentially bounded mappings...569

$$
\begin{aligned}
& =\max \left\{d\left(\phi_{i}, \phi_{j}\right), d\left(\phi_{i}, T \phi_{i}\right), d\left(\phi_{j}, T \phi_{j}\right), \frac{d\left(\phi_{i}, T \phi_{j}\right)+d\left(\phi_{j}, T \phi_{i}\right)}{2}\right\} \\
& =\max \left\{d\left(\phi_{i}, \phi_{j}\right), d\left(\phi_{i}, \phi_{i-1}\right), d\left(\phi_{j}, \phi_{j-1}\right), \frac{d\left(\phi_{i}, \phi_{j-1}\right)+d\left(\phi_{j}, \phi_{i-1}\right)}{2}\right\} \\
& =\max \left\{\phi_{i}-\phi_{j}, \frac{\phi_{i}+\phi_{i-1}-\phi_{j}-\phi_{j-1}}{2}\right\} \\
& =\phi_{i}-\phi_{j} \\
& =\frac{i(i+1)(i+2)-j(j+1)(j+2)}{3} \\
& =\frac{(i-j)\left(i^{2}+j^{2}+i j+3(i+j)+2\right)}{3} .
\end{aligned}
$$

It yields that

$$
\begin{aligned}
\frac{d(T x, T y) e^{d(T x, T y)-M_{d}(x, y)}}{M_{d}(x, y)} & =\frac{d\left(T \phi_{i}, T \phi_{j}\right) e^{d\left(T \phi_{i}, T \phi_{j}\right)-M_{d}\left(\phi_{i}, \phi_{j}\right)}}{M_{d}\left(\phi_{i}, \phi_{j}\right)} \\
& =\frac{\left(i^{2}+j^{2}+i j-1\right)}{\left(i^{2}+j^{2}+i j+3(i+j)+2\right)} e^{\frac{(i-j)(-3(i+j)-3)}{3}} \\
& =\frac{\left(i^{2}+j^{2}+i j-1\right)}{\left(i^{2}+j^{2}+i j+3(i+j)+2\right)} e^{(j-i)(i+j+1)} \\
& \leq e^{6(j-i)} \\
& \leq e^{-6} .
\end{aligned}
$$

If $i>1$ and $j=1$, then

$$
\begin{aligned}
d(T x, T y) & =d\left(T \phi_{i}, T \phi_{1}\right) \\
& =d\left(\phi_{i-1}, 1\right) \\
& =\frac{(i-1) i(i+1)-3}{3}
\end{aligned}
$$

and

$$
\begin{aligned}
M_{d}(x, y) & =M_{d}\left(\phi_{i}, \phi_{1}\right) \\
& =\max \left\{d\left(\phi_{i}, \phi_{1}\right), d\left(\phi_{i}, T \phi_{i}\right), d\left(\phi_{1}, T \phi_{1}\right), \frac{d\left(\phi_{i}, T \phi_{1}\right)+d\left(\phi_{1}, T \phi_{i}\right)}{2}\right\} \\
& =\max \left\{d\left(\phi_{i}, \phi_{1}\right), d\left(\phi_{i}, \phi_{i-1}\right), d\left(\phi_{1}, 1\right), \frac{d\left(\phi_{i}, 1\right)+d\left(\phi_{1}, \phi_{i-1}\right)}{2}\right\}
\end{aligned}
$$




$$
\begin{aligned}
& =d\left(\phi_{i}, \phi_{1}\right) \\
& =\frac{i(i+1)(i+2)-6}{3} .
\end{aligned}
$$

This follows that

$$
\begin{aligned}
\frac{d(T x, T y) e^{d(T x, T y)-M_{d}(x, y)}}{M_{d}(x, y)} & =\frac{d\left(T \phi_{i}, T \phi_{1}\right) e^{d\left(T \phi_{i}, T \phi_{1}\right)-M_{d}\left(\phi_{i}, \phi_{1}\right)}}{M_{d}\left(\phi_{i}, \phi_{1}\right)} \\
& =\frac{(i-1) i(i+1)-3}{i(i+1)(i+2)-6} e^{\frac{-3 i(i+1)+3}{3}} \\
& =\frac{(i-1) i(i+1)-3}{i(i+1)(i+2)-6} e^{-i(i+1)+1} \\
& <e^{-6}
\end{aligned}
$$

Case II: We consider $(x, y)=\left(\phi_{k}, 1\right)$ for all $k \in \mathbf{N} \backslash\{1\}$. Thus,

$$
\begin{aligned}
d(T x, T y) & =d\left(T \phi_{k}, T 1\right) \\
& =d\left(\phi_{k-1}, 1\right) \\
& =\frac{(k-1) k(k+1)-3}{3}
\end{aligned}
$$

and

$$
\begin{aligned}
M_{d}(x, y) & =M_{d}\left(\phi_{k}, 1\right) \\
& =\max \left\{d\left(\phi_{k}, 1\right), d\left(\phi_{k}, T \phi_{k}\right), d(1, T 1), \frac{d\left(\phi_{k}, T 1\right)+d\left(1, T \phi_{k}\right)}{2}\right\} \\
& =\max \left\{d\left(\phi_{k}, 1\right), d\left(\phi_{k}, \phi_{k-1}\right), d(1,1), \frac{d\left(\phi_{k}, 1\right)+d\left(1, \phi_{k-1}\right)}{2}\right\} \\
& =d\left(\phi_{k}, 1\right) \\
& =\frac{k(k+1)(k+2)-3}{3} .
\end{aligned}
$$

This implies that

$$
\begin{aligned}
\frac{d(T x, T y) e^{d(T x, T y)-M_{d}(x, y)}}{M_{d}(x, y)} & =\frac{d\left(T \phi_{k}, T 1\right) e^{d\left(T \phi_{k}, T 1\right)-M_{d}\left(\phi_{k}, 1\right)}}{M_{d}\left(\phi_{k}, 1\right)} \\
& =\frac{(k-1) k(k+1)-3}{k(k+1)(k+2)-3} e^{-k(k+1)} \\
& <e^{-6}
\end{aligned}
$$




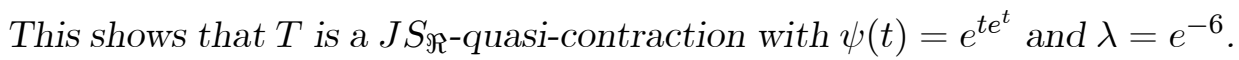
Moreover, there exists $x_{0}=\phi_{2} \in X$ such that $\left(\phi_{2}, T \phi_{2}\right)=\left(\phi_{2}, \phi_{1}\right) \in \Re$. This shows that $X(T ; \Re)$ is a nonempty set. Therefore, all conditions of Theorem 3.2 are satisfied and so we get the existence of a fixed point of $T$. In this case, $T$ has infinite fixed points (see Figure 1).

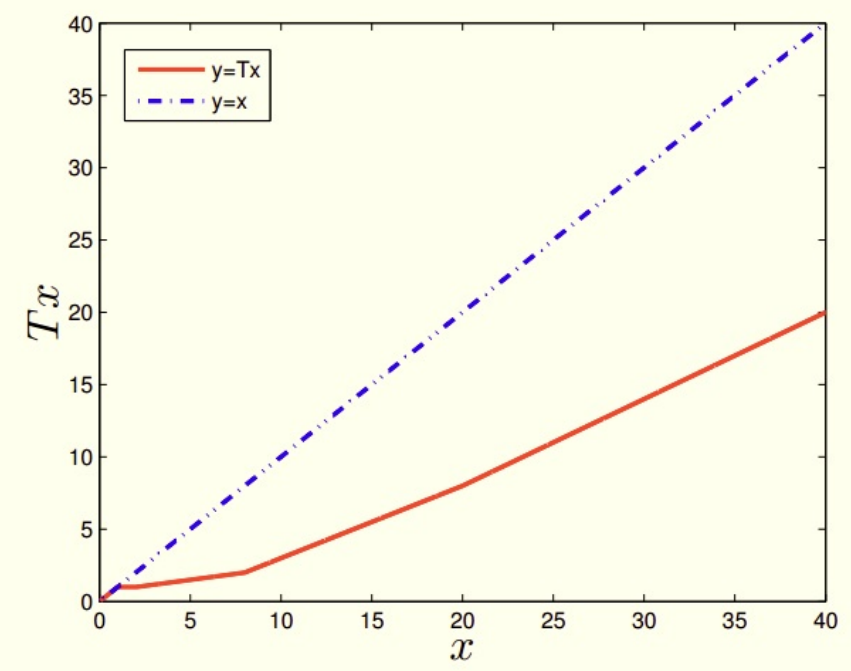

Figure 1: Graphs of $y=x$ and $y=T x$ in Example 3.3.

Remark 3.4. Note that Li and Jiang's result in [14] is not applicable in Example 3.3 since the $L i$ and Jiang's $J S$-quasi-contractive condition (2.1) with $\psi \in \Psi$ does not hold for each $x, y \in[0,1)$.

In the following result, we avoid the continuity of $T$.

Theorem 3.5. Theorem 3.2 also holds if we replace the hypothesis $(d)$ by the following one:

$\left(d^{\prime}\right)(x, d)$ is $\Re$-nondecreasing-regular.

Proof. The arguments of the proof of Theorem 3.2 prove that there exist the sequence $\left\{x_{n}\right\} \subseteq X$ and a point $x^{*} \in X$ such that $x_{n} \rightarrow x^{*}$ as $n \rightarrow \infty$. Since $\left(x_{n}, x_{n+1}\right) \in \Re$ for all $n \in \mathbf{N}$, then $\left(x_{n}, x^{*}\right) \in \Re$ for all $n \in \mathbf{N}$. We consider two cases depending on $P=\left\{n \in \mathbf{N}: T x_{n}=T x^{*}\right\}$. 
Case I: If $P$ is finite, then there is $n_{0} \in \mathbf{N}$ such that $T x_{n} \neq T x^{*}$ for all $n \geq n_{0}$. In particular, $x_{n} \neq x^{*}, d\left(x_{n}, x^{*}\right)>0$ and $d\left(T x_{n}, T x^{*}\right)>0$ for all $n \geq n_{0}$. Then

$$
\begin{aligned}
\psi\left(d\left(x_{n+1}, T x^{*}\right)\right) & =\psi\left(d\left(T x_{n}, T x^{*}\right)\right) \\
& \leq\left[\psi\left(M_{d}\left(x_{n}, x^{*}\right)\right)\right]^{\lambda} \\
& =\left[\psi\left(\max \left\{d\left(x_{n}, x^{*}\right), d\left(x_{n}, x_{n+1}\right), d\left(x^{*}, T x^{*}\right), \frac{d\left(x_{n}, T x^{*}\right)+d\left(x^{*}, T x_{n}\right)}{2}\right\}\right)\right]^{\lambda}
\end{aligned}
$$

for all $n \geq n_{0}$. Now, we will assume that $x^{*}$ is not a fixed point of $T$, that is, $d\left(x^{*}, T x^{*}\right)>0$. Letting $n \rightarrow \infty$ in (3.19), by the continuity of $d$ and $\psi$ we obtain

$$
\begin{aligned}
\psi\left(d\left(x^{*}, T x^{*}\right)\right) & =\lim _{n \rightarrow \infty} \psi\left(d\left(x_{n+1}, T x^{*}\right)\right) \\
& \leq \lim _{n \rightarrow \infty}\left[\psi\left(d\left(x^{*}, T x^{*}\right)\right)\right]^{\lambda} \\
& =\left[\psi\left(d\left(x^{*}, T x^{*}\right)\right)\right]^{\lambda} \\
& <\psi\left(d\left(x^{*}, T x^{*}\right)\right),
\end{aligned}
$$

which is a contradiction. Therefore, $d\left(x^{*}, T x^{*}\right)=0$. This means that $x^{*}=T x^{*}$ and so $x^{*}$ is a fixed point of $T$.

Case II: If $P$ is not finite, there is a subsequence $\left\{x_{n_{k}}\right\}$ of $\left\{x_{n}\right\}$ such that $x_{n_{k}+1}=T x_{n_{k}}=T x^{*}$ for all $k \in \mathbf{N}$. Since $x_{n} \rightarrow x^{*}$, we get $T x^{*}=x^{*}$ and so $x^{*}$ is a fixed point of $T$.

In any case, $x^{*}$ is a fixed point of $T$.

The following theorem guarantees the uniqueness of the fixed point in Theorems 3.2 and 3.5.

Theorem 3.6. In addition to the hypothesis of Theorem 3.2 (respectively, Theorem 3.5), suppose that $\Upsilon(x, y, \Re)$ is nonempty for all $x, y \in * F i x(T)$. Then $T$ has a unique fixed point. 
Proof. To prove the uniqueness, assume that $x$ and $y$ are two distinct fixed points of $T$. Then $T x \neq T y$. Since $\Upsilon(x, y, \Re)$ is nonempty, there is a path $\left\{z_{0}, z_{1}, z_{2}, \ldots, z_{k}\right\}$ of some finite length $k$ in $\Re$ from $x$ to $y$. Then

$$
z_{0}=x, \quad z_{k}=y, \quad\left(z_{i}, z_{i+1}\right) \in \Re \quad \text { for each } i=0,1,2, \ldots, k-1 .
$$

Since $\Re$ is a transitive relation, we get

$$
\left(x, z_{1}\right) \in \Re,\left(z_{1}, z_{2}\right) \in \Re, \ldots,\left(z_{k-1}, y\right) \in \Re \quad \Rightarrow \quad(x, y) \in \Re .
$$

By a $J S_{\Re \text {-quasi-contractive condition, we get }}$

$$
\psi(d(x, y))=\psi(d(T x, T y)) \leq\left[\psi\left(M_{d}(x, y)\right)\right]^{\lambda},
$$

where $M_{d}(x, y)=\max \left\{d(x, y), \frac{d(x, y)+d(y, x)}{2}\right\}=d(x, y)$. Then

$$
\psi(d(x, y)) \leq[\psi(d(T x, T y))]^{\lambda}<\psi(d(x, y)),
$$

which is a contradiction. Therefore, $x=y$. This means that $T$ has a unique fixed point.

Remark 3.7. If we take $\Re$ is the universal relation on $X$ in Theorem 3.6, we get the main result of $\mathrm{Li}$ and Jiang [14] (see in Theorem 2.2 in this paper). So all of particular results Li and Jiang [14] can be obtained by our main results.

\section{Some multidimensional results}

In this section, we illustrate how to obtain multidimensional results from the described results in Section 3 by involving very simple tools. Let $X$ be a nonempty set and $N$ denote a positive integer. We will denote by $X^{N}$ the Cartesian product $\underbrace{X \times X \times \ldots \times X}_{N-\text { terms }}$.

Definition 4.1 ([7]). Let $X$ be a nonempty set and let $T: X^{N} \rightarrow X$ be a given mapping. An element $\left(x_{1}, x_{2}, \ldots, x_{N}\right) \in X^{N}$ is said to be a fixed point of $N$-order of the mapping $T$ if

$$
\begin{aligned}
& T\left(x_{1}, x_{2}, \ldots, x_{N}\right)=x_{1}, \\
& T\left(x_{2}, x_{3}, \ldots, x_{N}, x_{1}\right)=x_{2}, \\
& \quad \vdots \\
& T\left(x_{N}, x_{1}, \ldots, x_{N-1}\right)=x_{N} .
\end{aligned}
$$


For convenience, we set

Fix $N(T):=\left\{z \in X^{N}: z\right.$ is a fixed point of $N$-order of $\left.T: X^{N} \rightarrow X\right\}$.

Given a binary relation $\Re$ on $X$, we will denote by $\Re^{N}$ the binary relation on the product space $X^{N}$ defined by

$\left(\left(x_{1}, x_{2}, \ldots, x_{N}\right),\left(y_{1}, y_{2}, \ldots, y_{N}\right)\right) \in \Re^{N} \quad \Leftrightarrow \quad\left(x_{1}, y_{1}\right) \in \Re,\left(x_{2}, y_{2}\right) \in \Re, \ldots,\left(x_{N}, y_{N}\right) \in \Re$.

If $T: X^{N} \rightarrow X$ is a mapping, let us denote by $X^{N}\left(T ; \Re^{N}\right)$ the set of all points $\left(x_{1}, x_{2}, \ldots, x_{N}\right) \in X^{N}$ such that

$\left(\left(x_{1}, x_{2}, \ldots, x_{N}\right),\left(T\left(x_{1}, x_{2}, \ldots, x_{N}\right), T\left(x_{2}, x_{3}, \ldots, x_{N}, x_{1}\right), \ldots, T\left(x_{N}, x_{1}, \ldots, x_{N-1}\right)\right)\right) \in \Re^{N}$,

that is,

$$
\left(x_{i}, T\left(x_{i}, x_{i+1}, \ldots, x_{N}, x_{1}, x_{2}, \ldots, x_{i-1}\right)\right) \in \Re \quad \text { for each } i \in\{1,2, \ldots, N\} .
$$

Definition 4.2. If $N \geq 2$ and $T: X^{N} \rightarrow X$ is a mapping, a binary relation $\Re$ on $X$ is called $T_{N}$-closed if for any $\left(x_{1}, x_{2}, \ldots, x_{N}\right),\left(y_{1}, y_{2}, \ldots, y_{N}\right) \in X^{N}$,

$$
\left\{\begin{array}{c}
\left(x_{1}, y_{1}\right) \in \Re, \\
\left(x_{2}, y_{2}\right) \in \Re, \\
\vdots \\
\left(x_{N}, y_{N}\right) \in \Re
\end{array}\right\} \Rightarrow\left\{\begin{array}{c}
\left(T\left(x_{1}, x_{2}, \ldots, x_{N}\right), T\left(y_{1}, y_{2}, \ldots, y_{N}\right)\right) \in \Re, \\
\left(T\left(x_{2}, x_{3}, \ldots, x_{N}, x_{1}\right), T\left(y_{2}, y_{3}, \ldots, y_{N}, y_{1}\right)\right) \in \Re, \\
\vdots \\
\left(T\left(x_{N}, x_{1}, \ldots, x_{N-1}\right), T\left(y_{N}, y_{1}, \ldots, y_{N-1}\right)\right) \in \Re
\end{array}\right\} .
$$

Let us denote by $G_{T}^{N}: X^{N} \rightarrow X^{N}$ the mapping

$G_{T}^{N}\left(x_{1}, x_{2}, \ldots, x_{N}\right)=\left(T\left(x_{1}, x_{2}, \ldots, x_{N}\right), T\left(x_{2}, x_{3}, \ldots, x_{N}, x_{1}\right), \ldots, T\left(x_{N}, x_{1}, \ldots, x_{N-1}\right)\right)$.

The following results guarantee that multidimensional notions can be interpreted in terms of $G_{T}^{N}$.

Lemma 4.3. Given $N \geq 2$ and $T: X^{N} \rightarrow X$, a point $\left(x_{1}, x_{2}, \ldots, x_{N}\right) \in$ $X^{N}$ is a fixed point of $N$-order of the mapping $T$ if and only if it is a fixed point of $G_{T}^{N}$.

Lemma 4.4. Given $N \geq 2$ and $T: X^{N} \rightarrow X$, a binary relation $\Re$ defined on $X$ is $T_{N}$-closed if and only if the binary relation $\Re^{N}$ defined on $X^{N}$ is $G_{T}^{N}$-closed. 
Lemma 4.5. Given $N \geq 2$ and $T: X^{N} \rightarrow X$, a point $\left(x_{1}, x_{2}, \ldots, x_{N}\right) \in$ $X^{N}\left(T ; \Re^{N}\right)$ if and only if $\left(x_{1}, x_{2}, \ldots, x_{N}\right) \in X^{N}\left(G_{T}^{N} ; \Re^{N}\right)$.

Lemma 4.6. Let $(X, d)$ be a metric space. Consider the product space $X^{N}$. Suppose that $D^{N}: X^{N} \times X^{N} \rightarrow \mathbf{R}$ is given by

$$
D^{N}(A, B)=\sum_{i=1}^{N} d\left(a_{i}, b_{i}\right)
$$

for all $A=\left(a_{1}, a_{2}, \ldots, a_{N}\right), B=\left(b_{1}, b_{2}, \ldots b_{N}\right) \in X^{N}$. Then the following properties hold.

1. $\left(X^{N}, D^{N}\right)$ is also a metric space.

2. Let $\left\{A_{n}=\left(a_{n}^{1}, a_{n}^{2}, \ldots a_{n}^{N}\right)\right\}$ be a sequence on $X^{N}$ and let $A=\left(a_{1}, a_{2}, \ldots, a_{N}\right) \in$ $X^{N}$. Then $A_{n} D^{N} \longrightarrow A$ if and only if $a_{n}^{i} d \longrightarrow a_{i}$ for all $i \in\{1,2, \ldots, N\}$.

3. If $\left\{A_{n}=\left(a_{n}^{1}, a_{n}^{2}, \ldots a_{n}^{N}\right)\right\}$ is a sequence on $X^{N}$, then $\left\{A_{n}\right\}$ is $D^{N_{-}}$ Cauchy if and only if $\left\{a_{n}^{i}\right\}$ is Cauchy for all $i \in\{1,2, \ldots, N\}$.

4. $(X, d)$ is complete if and only if $\left(X^{N}, D^{N}\right)$ is complete.

Definition 4.7. Let $X$ be a nonempty set and let $\Re$ be a binary relation on $X$. For $\left(x_{1}, x_{2}, \ldots, x_{N}\right),\left(y_{1}, y_{2}, \ldots, y_{N}\right) \in X^{N}$, a path of length $k$ in $\Re^{N}$ from $\left(x_{1}, x_{2}, \ldots, x_{N}\right)$ to $\left(y_{1}, y_{2}, \ldots, y_{N}\right)$ is a finite sequence $\left\{\left(z_{0}^{1}, z_{0}^{2}, \ldots, z_{0}^{N}\right),\left(z_{1}^{1}, z_{1}^{2}, \ldots, z_{1}^{N}\right), \ldots,\left(z_{k}^{1}, z_{k}^{2}, \ldots, z_{k}^{N}\right)\right\} \subseteq X^{N}$ satisfying the following conditions:

(i) $\left(z_{0}^{1}, z_{0}^{2}, \ldots, z_{0}^{N}\right)=\left(x_{1}, x_{2}, \ldots, x_{N}\right)$ and $\left(z_{k}^{1}, z_{k}^{2}, \ldots, z_{k}^{N}\right)=\left(y_{1}, y_{2}, \ldots, y_{N}\right)$;

(ii) $\left(\left(z_{i}^{1}, z_{i}^{2}, \ldots, z_{i}^{N}\right),\left(z_{i+1}^{1}, z_{i+1}^{2}, \ldots, z_{i+1}^{N}\right)\right) \in \Re^{N}$ for all $i=0,1,2, \ldots, k-1$.

We denote by $\Upsilon\left(\left(x_{1}, x_{2}, \ldots, x_{N}\right),\left(y_{1}, y_{2}, \ldots, y_{N}\right), \Re^{N}\right)$ the class of all paths in $\Re^{N}$ from $\left(x_{1}, x_{2}, \ldots, x_{N}\right)$ to $\left(y_{1}, y_{2}, \ldots, y_{N}\right)$.

Now, we introduce the notion of a $J S_{\Re^{N}}$-quasi-contraction for $N \geq 2$.

Definition 4.8. Let $\Re$ be a binary relation on a metric space $(X, d)$ and $T: X^{N} \rightarrow X$ a mapping. Given

$Z^{N}=\left\{\begin{array}{c}\left(\left(x_{1}, x_{2}, \ldots, x_{N}\right),\left(y_{1}, y_{2}, \ldots, y_{N}\right)\right) \in \Re^{N}: \\ T\left(x_{i}, x_{i+1}, \ldots, x_{N}, x_{1}, x_{2}, \ldots, x_{i-1}\right) \neq T\left(y_{i}, y_{i+1}, \ldots, y_{N}, y_{1}, y_{2}, \ldots, y_{i-1}\right) \\ \text { for all } i \in\{1,2, \ldots, N\}\end{array}\right\}$, 
we say that $T$ is a $J S_{\Re^{N}}$-quasi-contraction if there exist $\psi:(0, \infty) \rightarrow(1, \infty)$ and $\lambda \in(0,1)$ such that

$\psi\left(\sum_{i=1}^{N} d\left(T\left(x_{i}, x_{i+1}, \ldots, x_{N}, x_{1}, x_{2}, \ldots, x_{i-1}\right), T\left(y_{i}, y_{i+1}, \ldots, y_{N}, y_{1}, y_{2}, \ldots, y_{i-1}\right)\right),\right)$ $\leq\left[\psi\left(M_{\sum_{i=1}^{N} d}\left(x_{i}, y_{i}\right)\right)\right]^{\lambda}$

for all $\left(\left(x_{1}, x_{2}, \ldots, x_{N}\right),\left(y_{1}, y_{2}, \ldots, y_{N}\right)\right) \in \mathcal{Z}^{N}$, where

$$
=\max \left\{\begin{array}{c}
M_{\sum_{i=1}^{N} d\left(x_{i}, y_{i}\right)=}\left\{\begin{array}{c}
\sum_{i=1}^{N} d\left(x_{i}, y_{i}\right), \sum_{i=1}^{N} d\left(x_{i}, T\left(x_{i}, x_{i+1}, \ldots, x_{N}, x_{1}, x_{2}, \ldots, x_{i-1}\right)\right), \\
\sum_{i=1}^{N} d\left(y_{i}, T\left(y_{i}, y_{i+1}, \ldots, y_{N}, y_{1}, y_{2}, \ldots, y_{i-1}\right)\right) \\
\frac{\sum_{i=1}^{N} d\left(x_{i}, T\left(y_{i}, y_{i+1}, \ldots, y_{N}, y_{1}, y_{2}, \ldots, y_{i-1}\right)\right)+\sum_{i=1}^{N} d\left(y_{i}, T\left(x_{i}, x_{i+1}, \ldots, x_{N}, x_{1}, x_{2}, \ldots, x_{i-1}\right)\right)}{2}
\end{array}\right\} .
\end{array}\right.
$$

Next, we show how to use Theorem 3.2 in order to deduce multidimensional fixed point results that guarantee the existence of fixed points of $N$-order.

Theorem 4.9. Let $(X, d)$ be a complete metric space, $\Re$ a transitive relation on $X$, and $T: X^{N} \rightarrow X$ a mapping. Suppose that the following conditions hold:

(a) $X^{N}\left(T ; \Re^{N}\right)$ is nonempty,

(b) $\Re$ is $T_{N}$-closed,

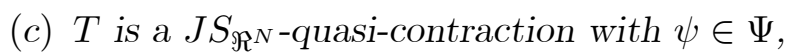

(d) $T$ is continuous.

Then $T$ has a fixed point of $N$-order.

Proof. By items 1 and 4 of Lemma 4.6, $\left(X^{N}, D^{N}\right)$ is a complete metric space. By Lemma 4.4, the binary relation $\Re^{N}$ defined on $X^{N}$ is $G_{T}^{N \text { - }}$ closed. Assume that $\left(x_{0}^{1}, x_{0}^{2}, \ldots, x_{0}^{N}\right) \in X^{N}\left(T ; \Re^{N}\right)$. By Lemma 4.5 we get $\left(x_{0}^{1}, x_{0}^{2}, \ldots, x_{0}^{N}\right) \in X^{N}\left(G_{T}^{N} ; \Re^{N}\right)$. Since $T$ is continuous, we have $G_{T}^{N}$ is also continuous. Now, we will show that $G_{T}^{N}$ is a $J S_{\Re^{N}}$-quasi-contraction with $\psi \in \Psi$. Let $A=\left(x_{1}, x_{2}, \ldots, x_{N}\right), B=\left(x_{1}, x_{2}, \ldots, x_{N}\right) \in \Re^{N}$ such that $G_{T}^{N} A \neq G_{T}^{N} B$. This implies that $T\left(x_{i}, x_{i+1}, \ldots, x_{N}, x_{1}, x_{2}, \ldots, x_{i-1}\right) \neq$ 
$T\left(y_{i}, y_{i+1}, \ldots, y_{N}, y_{1}, y_{2}, \ldots, y_{i-1}\right)$ for all $i \in\{1,2, \ldots, N\}$ and hence $(A, B) \in$ $\mathcal{Z}^{N}$. Since $T$ is a $J S_{\Re^{N}}$-quasi-contraction mapping, we obtain

$$
\begin{aligned}
\psi & \left.D^{N}\left(G_{T}^{N} A, G_{T}^{N} B\right)\right)=\psi\left(D^{N}\left(G_{T}^{N}\left(x_{1}, x_{2}, \ldots, x_{N}\right), G_{T}^{N}\left(x_{1}, x_{2}, \ldots, x_{N}\right)\right)\right) \\
& =\psi\left(D^{N}\left(\begin{array}{c}
\left(T\left(x_{1}, x_{2}, \ldots, x_{N}\right), T\left(x_{2}, x_{3}, \ldots, x_{N}, x_{1}\right), \ldots, T\left(x_{N}, x_{1}, \ldots, x_{N-1}\right)\right), \\
\left(T\left(y_{1}, y_{2}, \ldots, y_{N}\right), T\left(y_{2}, y_{3}, \ldots, y_{N}, y_{1}\right), \ldots, T\left(y_{N}, y_{1}, \ldots, y_{N-1}\right)\right)
\end{array}\right)\right) \\
& =\psi\left(\sum_{i=1}^{N} d\left(T\left(x_{i}, x_{i+1}, \ldots, x_{N}, x_{1}, x_{2}, \ldots, x_{i-1}\right), T\left(y_{i}, y_{i+1}, \ldots, y_{N}, y_{1}, y_{2}, \ldots, y_{i-1}\right)\right),\right) \\
& \leq\left[\psi\left(M_{\sum_{i=1}^{N} d}\left(x_{i}, y_{i}\right)\right)\right]^{\lambda} \\
& =\left[\psi\left(\max \left\{\begin{array}{c}
\sum_{i=1}^{N} d\left(x_{i}, y_{i}\right), \sum_{i=1}^{N} d\left(x_{i}, T\left(x_{i}, x_{i+1}, \ldots, x_{N}, x_{1}, x_{2}, \ldots, x_{i-1}\right)\right), \\
\sum_{i=1}^{N} d\left(y_{i}, T\left(y_{i}, y_{i+1}, \ldots, y_{N}, y_{1}, y_{2}, \ldots, y_{i-1}\right)\right), \overline{2}
\end{array}\right)\right]^{\lambda}\right. \\
& =\left[\psi\left(\max \left\{D^{N}(A, B), D^{N}\left(A, G_{T}^{N} A\right), D^{N}\left(B, G_{T}^{N} B\right), \frac{D^{N}\left(A, G_{T}^{N} B\right)+D^{N}\left(B, G_{T}^{N} A\right)}{2}\right\}\right)\right]^{\lambda} \\
& =\left[\psi\left(M_{D^{N}}(A, B)\right)\right]^{\lambda} .
\end{aligned}
$$

Applying Theorem 3.2, there exists $\hat{X}=\left(\hat{x_{1}}, \hat{x_{2}}, \ldots, \hat{x_{N}}\right) \in X^{N}$ such that $G_{T}^{N}(\hat{X})=\hat{X}$, that is, $\left(\hat{x_{1}}, \hat{x_{2}}, \ldots, \hat{x_{N}}\right)$ is a fixed point of $G_{T}^{N}$. Using Lemma $4.3,\left(\hat{x_{1}}, \hat{x_{2}}, \ldots, \hat{x_{N}}\right)$ is a fixed point of $N$-order of $T$. This completes the proof.

By using Theorem 3.5 with a similar technique as in the proof of Theorem 4.9, we get the following existence result of a fixed point of $N$-order.

Theorem 4.10. Let $(X, d)$ be a complete metric space, $\Re$ a transitive relation on $X$, and $T: X^{N} \rightarrow X$ a mapping. Suppose that the following conditions hold:

(a) $X^{N}\left(T ; \Re^{N}\right)$ is nonempty,

(b) $\Re$ is $T_{N}$-closed,

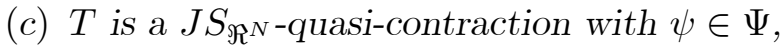

(d) $X^{N}$ is $\Re^{N}$-nondecreasing-regular with the metric $D^{N}$.

Then $T$ has a fixed point of $N$-order.

By using Theorem 3.6, we get the following uniqueness result of fixed point of $N$-order. 
Theorem 4.11. In addition to the hypothesis of Theorem 4.9 (respectively, Theorem 4.10), suppose that $\Upsilon\left(\left(x_{1}, x_{2}, \ldots, x_{N}\right),\left(y_{1}, y_{2}, \ldots, y_{N}\right), \Re^{N}\right)$ is nonempty for each $\left(x_{1}, x_{2}, \ldots, x_{N}\right),\left(y_{1}, y_{2}, \ldots, y_{N}\right) \in$ Fix $N(T)$. Then $T$ has a unique fixed point of $N$-order.

\section{Conclusion and Suggestion}

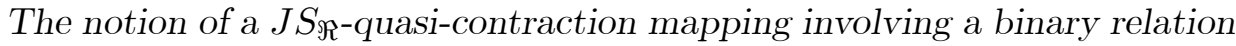
$\Re$ on its domain was first presented in this paper. New fixed point results for such contractions in a complete metric space endowed with a transitive relation are investigated. Many fixed point results can be obtained from our main results by setting some suitable relation and some control function involving the contractive condition. For instance, fixed point results of $\mathrm{Li}$ and Jiang [14] are special case of our main results. An example showing the real proper generalization of our main results is given. Furthermore, fixed point of $N$-order results can be derived from our fixed point results for a uni-dimaensional mapping. Finally, we give some suggestion to the reader for further investigation. Based on the utility of a relation concernig our fixed point results, the reader can be applied it together with the Thompson metric to solve the following nonlinear matrix equation:

$$
X^{r}=Q+\sum_{i=1}^{m} A_{i}^{*} \mathcal{G}_{i}(X) A_{i},
$$

where $r \geq 1, Q$ is a Hermitian positive definite matrix, $A_{i}$ is an $n \times n$ nonsingular matrix for all $i=1,2,3, \ldots, m$, and $\mathcal{G}_{i}$ is a continuous order preserving self-mapping on the set of all $n \times n$ positive definite matrices for all $i=1,2,3, \ldots, m$. We give the following question as the open problem to the reader

- Can we find the suitable condition which can be guaranteed the existence of a solution of the matrix equation (5.1)?

\section{Acknowledgement}

This study was supported by Thammasat University Research Fund, Contract No. TUGG 37/2562. The second author gratefully acknowledge the financial support provided by Faculty of Science and Technology, Contract No. SciGR3/2563. 


\section{References}

[1] S. Banach, "Sur les óperations dans les ensembles abstraits et leurs applications aux équations intégrales", Fundamenta mathematicae, vol. 3 , no. 1, pp. 133-181, 1922. [On line]. Available: https://bit.ly/2XmuBvp

[2] A. C. M. Ran and M. C. B. Reurings, "A fixed point theorem in partially ordered sets and some applications to matrix equations", Proceedings of the American Mathematical Society, vol. 132, no. 05, pp. 1435-1444, May 2004, doi: 10.1090/S0002-9939-03-07220-4

[3] A. Alam and M. Imdad, "Relation-theoretic contraction principle", Journal of fixed point theory and applications, vol. 17, no. 4, pp. 693-702, Jul. 2015, doi: 10.1007/s11784-015-0247-y

[4] D. Guo and V. Lakshmikantham, "Coupled fixed points of nonlinear operators with applications", Nonlinear analysis: theory, methods \& applications, vol. 11, no. 5, pp. 623-632, 1987, doi: 10.1016/0362546X(87)90077-0

[5] T. G. Bhaskar and V. Lakshmikantham, "Fixed point theorems in partially ordered metric spaces and applications", Nonlinear analysis: theory, methods \& applications, vol. 65, no. 7, pp. 1379-1393, Oct. 2006, doi: 10.1016/j.na.2005.10.017

[6] M. S. Asgari and B. Mousavi, "Coupled fixed point theorems with respect to binary relations in metric spaces", Journal of nonlinear sciences and applications, vol. 08, no. 02, pp. 153-162, Mar. 2015, doi: 10.22436/jnsa.008.02.07

[7] B. Samet and C. Vetro, "Coupled fixed point, F-invariant set and fixed point of N-order", Annals of functional analysis, vol. 1, no. 2, pp. 46-56, 2010, doi: $10.15352 / \mathrm{afa} / 1399900586$

[8] D. Wardowski, "Fixed points of a new type of contractive mappings in complete metric spaces", Fixed point theory and applications, vol. 2012, Art ID. 94, Jun. 2012, doi: 10.1186/1687-1812-2012-94

[9] F. Khojasteh, S. Shukla, and S. Radenovic, "A new approach to the study of fixed point theory for simulation functions", Filomat, vol. 29, no. 6, pp. 1189-1194, 2015., doi: 10.2298/FIL1506189K

[10] L. Ćirić, "Generalized contractions and fixed-point theorems", Publications de l'Institut Mathématique (Online), vol. 12, no. 26, pp.19-26, 1971, [On line]. Available: https://bit.ly/36njWEQ

[11] L. Ćirić, “A generalization of Banach's contraction principle”, Proceedings of the American Mathematical Society, vol. 45, no. 2, pp. 267-273, Aug. 1974, doi: 10.2307/2040075

[12] M. Jleli and B. Samet, "A new generalization of the Banach contraction principle", Journal of inequalities and applications, vol. 2014, no. 1, Art ID. 38, May 2014, doi: 10.1186/1029-242X-2014-38 
[13] N. Hussain, V. Parvaneh, B. Samet, and C. Vetro, "Some fixed point theorems for generalized contractive mappings in complete metric spaces", Fixed point theory and applications, vol. 2015, no. 1, Art ID. 185, Oct. 2015, doi: 10.1186/s13663-015-0433-z.

[14] Z. Li and S. Jiang, "Fixed point theorems of JS-quasi-contractions", Fixed point theory and applications, vol. 2016, no. 1, Art ID. 40, Mar. 2016, doi: 10.1186/s13663-016-0526-3

[15] S. Lipschutz, Theory and problems of set theory and related topics. New York, NY: McGraw-Hill, 1964.

[16] B. C. Kolman, R. C. Busby, and S. C. Ross, Discrete mathematical structures, 3rd ed. New Delhi: Prentice Hall of India Pvt. Ltd, 2000.

[17] A. F. Roldán López de Hierro, "A unified version of Ran and Reuring's theorem and Nieto and Rodríguez-Lopez's theorem and low-dimensional generalizations", Applied mathematics \& information sciences, vol. 10, no. 2, pp. 383-393, Mar. 2016, doi: 10.18576/amis/100201 